J. Phys. IV France 138 (2006) 171-179

(C) EDP Sciences, Les Ulis

DOI: 10.1051/jp4:2006138020

\title{
Absorptions UV dans le fonctionnement des sources lasers solides à ions de terres rares
}

\author{
R. Moncorgé ${ }^{1}$, J. Margerie ${ }^{1}$, J.L. Doualan ${ }^{1}$, P. Nagtegaele ${ }^{1}$, Y. Guyot ${ }^{2}$ \\ et M.F. Joubert ${ }^{2}$ \\ ${ }^{1}$ Centre Interdisciplinaire de Recherches lons Lasers (CIRIL), Équipe "Matériaux \\ et Instrumentation Laser » UMR 6637 CEA-CNRS-ENSICAEN, Université de Caen, \\ 6 Bd. Maréchal Juin, 14050 Caen, France \\ e-mail : richard.moncorge@ensicaen.fr \\ ${ }^{2}$ Laboratoire de Physico-Chimie des Matériaux Luminescents, UMR 5620 CNRS, \\ Université Claude Bernard de Lyon 1, 43 Bd. 11 Novembre 1918, 69622 Villeurbanne, France
}

\begin{abstract}
Résumé. L'article donne une description et analyse les phénomènes d'absorption UV à l'origine des mécanismes de solarisation et de variation d'indice de réfraction observés dans certains matériaux dopés terres rares étudiés pour leurs propriétés laser UV ou infrarouge lorsqu'ils sont soumis à de fortes densités d'excitation optique.
\end{abstract}

\section{INTRODUCTION}

De nombreux travaux ont été consacrés durant cette décennie à l'étude des mécanismes de pompage optique par étapes et aux mécanismes d'absorption dans les états excités (ESA) des ions de terres rares dans les matériaux solides pour les scintillateurs et les lasers [1-3]. Les résultats de ces recherches théoriques et expérimentales nous permettent aujourd'hui de mieux comprendre pourquoi il est si difficile de trouver un bon système laser solide fonctionnant dans le domaine UV et pourquoi, parmi ceux qui sont dopés par les ions $\mathrm{Ce}^{3+}, \mathrm{Pr}^{3+}$ et $\mathrm{Nd}^{3+}$, seuls quelques fluorures dopés par les ions $\mathrm{Ce}^{3+}$ ont conduit à des systèmes laser accordables et des amplificateurs réellement efficaces [4-6], pourquoi aucun matériau dopé $\mathrm{Pr}^{3+}$ n'a jamais fonctionné et pourquoi quelque espoir existe encore de voir fonctionner un cristal dopé $\mathrm{Nd}^{3+}$ dans le VUV suite à une excitation à deux photons.

D'un autre côté, la caractérisation et la compréhension de ces mécanismes de pompage optique multiphotonique et d'absorption dans les états excités aident grandement pour rendre compte des différences de polarisabilités des ions actifs dans leurs états fondamental et excités, différences qui semblent à l'origine du comportement auto-déclenché et de la formation des réseaux de diffraction transitoires observés dans des systèmes lasers fortement pompés tels que LiCAF:Cr [7-9] et YAG:Nd $[10,11]$.

Après une brève description des systèmes laser mentionnés plus haut et de leurs modes de fonctionnement particuliers, l'article portera sur les résultats spectroscopiques obtenus le plus récemment pour rendre compte par exemple des variations d'indice de réfraction spectaculaires de 5 à $7 \times 10^{-6}$ observés dans le YAG :Nd en présence d'un pompage diode intense et d'une excitation laser simultanée à $266 \mathrm{~nm}$ [11].

\section{ABSORPTION DANS L'ÉTAT EXCITÉ DES IONS DANS LES MATÉRIAUX ÉMETTEURS DE RAYONNEMENT UV}

Les matériaux solides susceptibles d'émettre un rayonnement laser dans l'ultraviolet sont essentiellement des cristaux à base de fluor (des fluorures) dont le domaine de transparence est typiquement d'une dizaine d'eV, dopés par les ions terres rares trivalents $\mathrm{Ce}^{3+}, \mathrm{Pr}^{3+}$ ou $\mathrm{Nd}^{3+}$. 
Les transitions optiques mis en jeu sont des transitions très intenses (dipolaires électriques) entre configurations électronique fondamentale et excitée $4 \mathrm{f}^{\mathrm{n}}$ et $4 \mathrm{f}^{\mathrm{n}-1} 5 \mathrm{~d}$ (voir Figure 1). Ce sont des transitions optiques de type vibronique, ce qui en fait potentiellement des systèmes lasers UV, voire VUV, très largement accordables au même titre que les autres systèmes laser vibroniques tels que les lasers à colorants, le Ti:Saphir ou plus récemment le Cr:ZnSe.

De fait, on est maintenant capable de fabriquer des systèmes laser solides à base d'ions $\mathrm{Ce}^{3+}$ que l'on peut pomper à l'aide de différentes sources, très largement accordables sur plusieurs dizaines de nm et très efficaces, le record ayant été obtenu avec le cristal LiLuF4: $\mathrm{Ce}^{3+}$ avec un rendement optique de l'ordre de $60 \%[4,5,12]$. Ces cristaux, notamment le $\mathrm{LiCAF}: \mathrm{Ce}^{3+}$, ont également servi d'amplificateurs pour des lasers UV nanosecondes de haute énergie $[13,14]$ et même pour des impulsions laser UV d'une centaine de femtosecondes et de quelques $\mathrm{mJ}[6]$.

Ceci étant, dans les domaines de longueur d'onde considérés, seuls les cristaux LiCaAlF6 et LiLuF4 dopés $\mathrm{Ce}^{3+}$ ont réellement donné des résultats intéressants. De plus, il n'a jamais été possible de « faire laser » un cristal dopé $\operatorname{Pr}^{3+}$, ce qui est regrettable car les domaines d'émission correspondants (220-300 nm) sont très intéressants. Enfin, seuls les systèmes $\mathrm{LaF}_{3}$ et $\mathrm{LiYF}_{4}$ dopés $\mathrm{Nd}^{3+}$ semblent avoir conduits à un effet laser, les résultats en question ayant été obtenus jusqu'à présent, dans chaque cas, par un seul groupe $[15,16]$.

Les possibilités offertes sont finalement assez restreintes. Les rendements très modestes voire l'absence de gain laser constatés dans la plupart de ces matériaux sont en fait la conséquence de deux types de pertes optiques : (i) la formation de centres pièges (absorbants) que l'on nomme parfois centres colorés résultant de défauts ou d'impuretés toujours plus ou moins présents dans les cristaux selon leur nature et leur méthode de fabrication, (ii) l'absorption dans l'état excité émetteur 5d du rayonnement de pompage optique comme du rayonnement laser vers la bande de conduction du matériau hôte (voir flèches vers le haut de la Figure 1). Si il est toujours difficile de répondre à la question des défauts à l'origine des centres pièges, de gros efforts ont été entrepris ces dernières années pour mieux comprendre les mécanismes à l'origine des pertes dans les états excités des ions, en particulier en déterminant de manière théorique et expérimentale la position relative de leurs niveaux $4 \mathrm{f}$ et $5 \mathrm{~d}$ par rapport aux bandes de valence et de conduction des cristaux hôtes.

Cette dernière question est maintenant bien comprise dans le cas du cristal $\mathrm{LiYF}_{4}$ qui est l'une des matrices laser les plus importantes. Un modèle semi-empirique [17] permet en effet de positionner le niveau fondamental de la configuration $4 \mathrm{f}^{\mathrm{n}}$ et le niveau le plus bas de la configuration excitée $4 \mathrm{f}^{\mathrm{n}-1} 5 \mathrm{~d}$ de tous les ions de terres rares trivalents par rapport aux bandes de valence et de conduction du réseau hôte. Selon ce modèle, il est possible de calculer l'énergie de liaison de la configuration $4 \mathrm{f}$ pour un ion de terre rare trivalent $X^{3+}$ en utilisant l'expression :

$$
E\left(4 f, X^{3+}\right)=I_{4 f}-E_{L}+\alpha_{R}\left(R_{X}-R_{Y}\right)
$$

où $I_{4 f}$ est le potentiel d'ionisation de la terre rare [18] considérée et $E_{L}$ représente l'effet électrostatique intrinsèque du réseau environnant au site de la terre rare (énergie reliée à l'énergie de Madelung). Le troisième terme représente en fait une correction de $E_{L}$ due à la distorsion induite par l'introduction de l'ion dopant $X^{3+} . R_{X}$ et $R_{Y}$ sont les rayons ioniques [19] des ions $X^{3+}$ et $Y^{3+}$ (l'ion auquel se substitue l'ion dopant $X^{3+}$ dans $\mathrm{LiYF}_{4}$ ) et $\alpha_{R}$ mesure la variation d'énergie de liaison par unité de variation de rayon ionique. On détermine ainsi la position du niveau fondamental de la configuration $4 \mathrm{f}^{\mathrm{n}}$ de tous les ions de terres rares trivalents à partir des quelques données disponibles dans la littérature [20-22] et en traitant $E_{L}$ et $\alpha_{R}$ comme des paramètres ajustables. Dans le cas de $\mathrm{LiYF}_{4}$, comme le montre la Figure 2, le meilleur ajustement des données expérimentales est obtenu pour $E_{L}=29.87 \mathrm{eV}$ et $\alpha_{R}=13.98 \mathrm{eV} / \mathrm{ug}$ es positions des niveaux d'énergie les plus bas de la première configuration électronique excitée $4 \mathrm{f}^{\mathrm{n}-1} 5 \mathrm{~d}$ sont déduits des spectres expérimentaux reportés dans les références [1-3, 23]. Ces positions peuvent être également obtenues de manière approximative en utilisant un formalisme semi-empirique développé récemment [24]. Ce formalisme tend à prouver que les positions en question restent à peu près constantes 


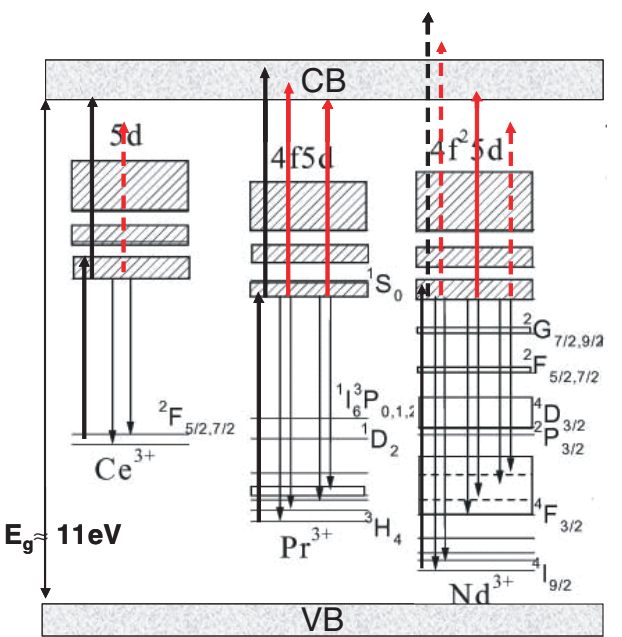

Figure 1. Niveaux d'énergie des ions $\mathrm{Ce}^{3+}, \mathrm{Pr}^{3+}$ et $\mathrm{Nd}^{3+}$ dans $\mathrm{LiYF}_{4}$ par rapport aux bandes de conduction et de valence du réseau hôte.

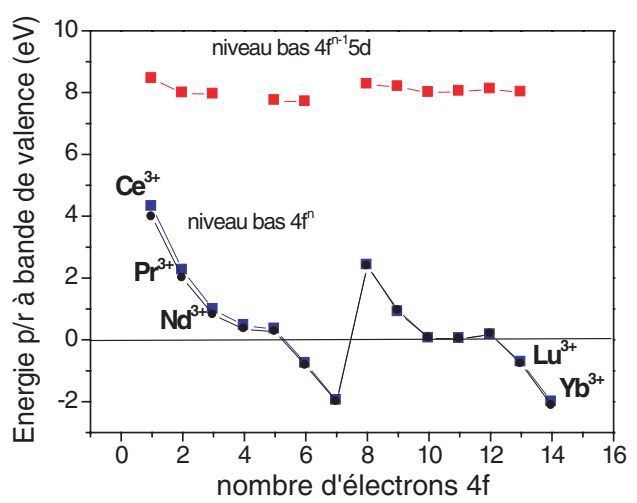

Figure 2. Niveaux d'énergie les plus bas des configurations électroniques $4 \mathrm{f}^{\mathrm{n}}$ et $4 \mathrm{f}^{\mathrm{n}-1} 5 \mathrm{~d}$ des ions de terres rares dans $\mathrm{LiYE}_{4}$.

par rapport aux bandes de valence et de conduction pour tous les ions de terres rares, ce qui est effectivement observé, à $0.5 \mathrm{eV}$ près.

Ceci étant, on peut ainsi positionner plus précisément l'ensemble des niveaux d'énergie des ions $\mathrm{Ce}^{3+}, \mathrm{Pr}^{3+}$ et $\mathrm{Nd}^{3+}$ par rapport aux bandes de valence et de conduction du matériau hôte $\mathrm{LiYF}_{4}$, ce qui est représenté sur la Figure 1, et comprendre un peu mieux ce qu'il advient lorsqu'on tente de pomper ces systèmes dans le proche UV voire dans l'UV du vide. Cela permet de constater que seuls les rayonnements émis par les ions $\mathrm{Ce}^{3+}$ et $\mathrm{Nd}^{3+}$ peuvent ne pas être absorbés dans l'état excité émetteur vers le bas de la bande de conduction. Ceci fournit donc une première explication au fait que seuls ces ions (jamais l'ion $\mathrm{Pr}^{3+}$ ) aient conduit à un effet laser. Ceci est renforcé par le fait que l'absorption du rayonnement de pompage optique dans l'état excité émetteur est également plus importante pour l'ion $\mathrm{Pr}^{3+}$ que pour les autres ions. On remarque aussi dans le cas de l'ion $\mathrm{Nd}^{3+}$ que seul le rayonnement de longueur d'onde intermédiaire semble être absorbé vers le bas de la bande de conduction et que le rayonnement de pompage optique pourrait ne pas l'être faute de niveaux disponibles, ce dernier aspect semblant confirmé par un calcul de bandes utilisant la méthode DFT [25] et montrant effectivement un minimum de densité d'état entre 2.5 et $6 \mathrm{eV}$ au dessus du plus bas niveau de la bande de conduction. Ces constations ouvrent donc la possibilité d'observer un effet laser dans $\mathrm{LiYF}_{4}: \mathrm{Nd}$ aux plus grandes longueurs d'onde, soit aux environs de $262 \mathrm{~nm}$ - ce qui a déjà été observé en pompant avec un laser moléculaire F2 à $157 \mathrm{~nm}$ [15], comme aux plus courtes, soit aux environs de $185 \mathrm{~nm}$, ce qui reste à prouver (mais qui a déjà été observé dans le cas du cristal $\mathrm{LaF}_{3}: \mathrm{Nd}^{3+}$ à une longueur d'onde de $172 \mathrm{~nm}[16])$.

A partir de là nous nous sommes intéressés à la possibilité de limiter les effets de pertes par absorption dans l'état excité, en particulier à la longueur d'onde de pompage optique, en étudiant des pompages par étapes à l'aide de photons de plus courtes longueurs d'onde, à priori moins néfastes du point de vue formation de centres colorés (solarisation). La question a été examinée plus particulièrement dans le cas des ions $\mathrm{Pr}^{3+}$ et $\mathrm{Nd}^{3+}$ en utilisant certains niveaux excités de la configuration électronique fondamentale $4 \mathrm{f}^{\mathrm{n}}$ comme niveaux intermédiaires. Deux photons bleus ou un photon rouge plus un photon bleu ont été considérés par exemple dans le cas de l'ion $\operatorname{Pr}^{3+}$ [26] en utilisant les niveaux ${ }^{3} \mathrm{P}_{0,1,2}$ ou le niveau ${ }^{1} \mathrm{D}_{2}$ comme niveaux intermédiaires, et deux photons bleus ou un photon rouge plus un photon violet ont été considérés dans le cas de $\mathrm{Nd}^{3+}$ [3] en passant par les niveaux ${ }^{4} \mathrm{D}_{3 / 2}$ et ${ }^{4} \mathrm{~F}_{3 / 2}$. 
Ces mécanismes ont d'abord été étudiés expérimentalement, en enregistrant des spectres d'absorption dans les différents états excités considérés, ceci à l'aide d'un montage pompe-sonde approprié [27], pour déterminer les longueurs d'onde et les polarisations des rayonnements présentant les sections efficaces d'absorption les plus favorables, puis d'un point de vue théorique, à l'aide de calculs de niveaux d'énergie et de simulations de spectres d'absorption, à partir de tous les niveaux métastables intéressants de la configuration $4 \mathrm{f}^{\mathrm{n}}$ [1-3]. Parmi les nombreux résultats importants obtenus dans ce cadre, il a été ainsi constaté, en accord avec l'expérience, une sorte d'effet mémoire de l'état de départ des transitions optiques. Cette effet mémoire, que nous avons mis en évidence dans le cas des ions $\mathrm{Pr}^{3+}$ et $\mathrm{Nd}^{3+}$ mais qui peut être généralisé à tous les autres ions de terres rares, se traduit en effet par un décalage vers le bleu du barycentre des bandes d'absorption avec l'énergie du niveau de départ [3]. Ce résultat nous a permis d'expliquer par exemple pourquoi il était préférable d'utiliser des photons rouge et violet et l'état intermédiaire ${ }^{4} \mathrm{~F}_{3 / 2}$ plutôt que deux photons bleus pour atteindre le bas de la bande $4 \mathrm{f}^{2} 5 \mathrm{~d}$ de l'ion $\mathrm{Nd}^{3+}$ dans LiYF4, un schéma d'excitation que nous tentons actuellement de mettre en œuvre à l'aide d'un montage où le même laser, un laser YAG :Nd (DIVA, Thales) doublé et quintuplé en fréquence associé à une cavité $\mathrm{Ti}: \mathrm{Sa}$, est utilisé pour pomper l'ion $\mathrm{Nd}^{3+}$ à 795 et à $213 \mathrm{~nm}$. Un premier essai a déjà été effectué, sans succès néanmoins en raison du dommage optique subis par le miroir dichroïque d'entrée et en raison de problèmes d'étanchéité, l'effet laser recherché se situant vers $185 \mathrm{~nm}$, donc à la limite du VUV.

\section{VARIATIONS D'INDICE DE RÉFRACTION ET DE POLARISABILITÉ DES IONS DANS LES MATÉRIAUX LASER SOUMIS À DE FORTES DENSITÉS D'EXCITATION}

Des variations d'indice de réfraction, autres que thermiques, ont été récemment observées et mises à profit dans certains systèmes laser de forte puissance pour construire ce qu'on appelle aujourd'hui des systèmes laser auto-organisés [11, 28, 29]. Il s'agit d'une problématique à laquelle nous nous intéressons actuellement car nous pensons que ces variations d'indice de réfraction athermiques sont probablement dues à des variations de polarisabilités des ions lorsqu'on les porte de leur état fondamental dans leurs états excités, un phénomène le plus souvent complètement négligé par les « laséristes » concernés dans leur description des effets non-linéaires induits (effets lentille et réseaux dynamiques).

Il a été démontré par exemple dans le cas du YAG :Nd par le groupe d'Antipov [11] à l'aide d'une expérience d'interférométrie transitoire qu'on pouvait provoquer des variations d'indice de réfraction très importantes de plus de $10^{-6}$ en pompant par diodes laser à $808 \mathrm{~nm}$ ou par lampe flash et que ces variations étaient probablement dues à des variations de polarisabilités des ions $\mathrm{Nd}^{3+}$ lorsqu' on les porte dans leur état métastable ${ }^{4} \mathrm{~F}_{3 / 2}$, dans le cas du pompage par diodes, mais aussi lorsqu'on les porte dans leurs autres états excités émetteurs ${ }^{4} \mathrm{D}_{3 / 2}$ et ${ }^{2} \mathrm{P}_{3 / 2}$ dans le domaine visible et ${ }^{2} \mathrm{~F}(2)_{5 / 2}$ dans le domaine UV, dans le cas d'un pompage flash (voir Figure 3).

Il a été démontré en outre, toujours avec le même montage d'interférométrie transitoire, en pompant cette fois simultanément à 808 et à $266 \mathrm{~nm}$ avec un laser YAG :Nd quadruplé en fréquence ce qui correspond à une absorption dans l'état excité très efficace du niveau métastable ${ }^{4} \mathrm{~F}_{3 / 2}$ vers la bande $4 \mathrm{f}^{2} 5 \mathrm{~d}$, que cette variation d'indice augmentait très rapidement, ceci pour des énergies UV relativement faibles de quelques $\mathrm{mJ} / \mathrm{cm}^{2}[11]$.

Notre objectif a donc été d'examiner la validité de cette hypothèse, d'abord en enregistrant certains spectres d'absorption dans l'état excité (ESA) et surtout en confrontant les données expérimentales aux estimations théoriques qui peuvent être faites des variations de polarisabilité $\Delta \alpha_{P}$ et des variations d'indice de réfraction $\Delta \mathrm{n}$, sachant que ces grandeurs peuvent être reliées entre elles, en faisant appel à la relation de Clausius-Mosotti, à l'aide de l'expression suivante :

$$
\Delta n=\left(\frac{2 \pi}{n} f_{L}^{2} \Delta \alpha_{P}\right) N_{e x}
$$


où $f_{L}=\frac{n^{2}+2}{3}$ représente le facteur de correction local de Lorentz habituel et $N_{\mathrm{ex}}$ la densité d'ions excités dans l'état considéré.

A partir de là, deux méthodes donnant des résultats quasiment identiques ont été utilisées. La première dite de l'oscillateur amorti permet d'obtenir la polarisabilité d'un ion dans un certain état électronique à une certaine fréquence à l'aide de la force d'oscillateur de la transition optique considérée, laquelle est déterminée théoriquement à partir des éléments de matrice dipolaires électriques correspondants. La deuxième méthode repose sur l'utilisation de la transformation de Kramers-Kronig qui permet de trouver l'expression de l'indice de réfraction à une certaine fréquence en fonction de la section efficace de la transition laquelle peut être également déterminée à partir des mêmes éléments de matrice que précédemment.

Cette étude nous a d'abord permis de vérifier le bon accord entre spectres expérimentaux et spectres théoriques mais aussi de trouver la relation de proportionnalité entre variations d'indice et population dans l'état excité. En supposant uniquement une excitation du niveau métastable émetteur ${ }^{4} \mathrm{~F}_{3 / 2}$, nous trouvons par exemple qu'à $1064 \mathrm{~nm}\left(9398 \mathrm{~cm}^{-1}\right)$, la longueur d'onde laser principale du YAG :Nd, la variation d'indice de réfraction est donnée par $\Delta n \approx 1.7 \times 10^{-25} N_{e x}$ et que la variation de polarisabilité correspondante est donnée par $\Delta \alpha_{P} \approx 1.6 \times 10^{-26} \mathrm{~cm}^{3}$.

En confrontant alors nos calculs avec les données de la littérature, les résultats d'interférométrie transitoire obtenus avec une pompe à $808 \mathrm{~nm}$ et une sonde à $633 \mathrm{~nm}$ [11] ou les résultats de mélange à 4 ondes obtenus à $514 \mathrm{~nm}$ [30], ce que nous avons reporté dans le Tableau suivant, nous constatons que ces résultats expérimentaux sont très proches de nos valeurs théoriques.

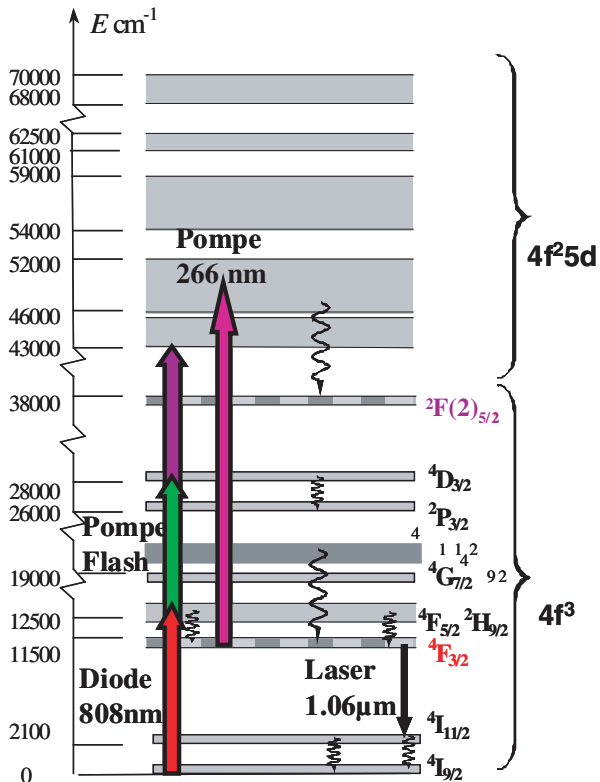

Figure 3. Niveaux d'énergie de l'ion $\mathrm{Nd}^{3+}$ dans YAG.

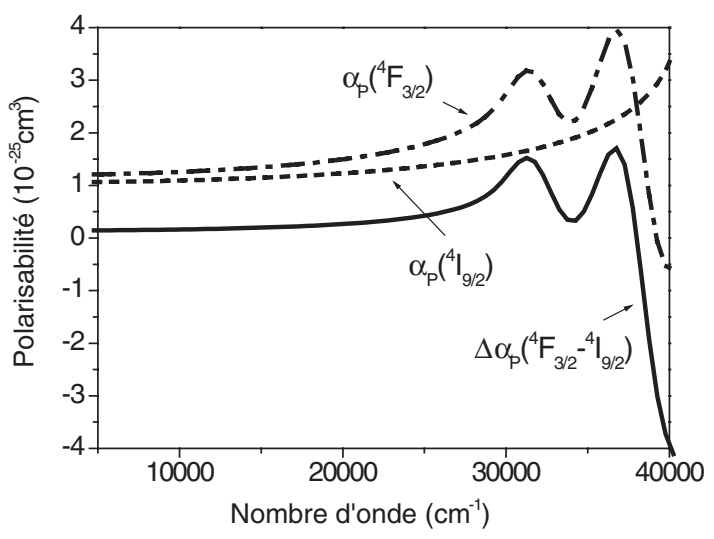

Figure 4. Courbes de polarisabilités associées aux niveaux foundamental et excité ${ }_{9 / 2}^{I}$ et $\frac{F}{3 / 2}$ et variations induites de l'infrarouge au proche UV.

Nous avons démontré que les variations de polarisabilité $\Delta \alpha_{2 \mathrm{~F}(2)-4 \mathrm{I}}$ associées à la population du niveau métastable ultraviolet ${ }^{2} \mathrm{~F}(2)_{5 / 2}$, en tenant compte de certains ajustements par rapport aux données reportées dans la référence [9] étaient vraiment d'un ordre de grandeur supérieures aux variations de polarisabilité $\Delta \alpha_{4 \mathrm{~F}-4 \mathrm{I}}$ associées au niveau métastable émetteur laser ${ }^{4} \mathrm{~F}_{3 / 2}$, ce qui explique, comme 
Tableau 1. Comparaison entre valeurs expérimentales et théoriques des variations de polarisabilitéde l'ion $\mathrm{Nd}^{3+}$ dans YAG lorsqu'on le porte de son état fondamental ${ }^{4} \mathrm{I}_{9 / 2}$ dans son état excité ${ }^{4} \mathrm{~F}_{3 / 2}$ (i.e. $\Delta \alpha_{4 \mathrm{~F}}-4 \mathrm{I}$ ) ou dans son état excité ${ }^{2} \mathrm{~F}(2)_{5 / 2}$ (i.e. $\Delta \alpha_{2 \mathrm{~F}(2)-4 \mathrm{I}}$ ).

\begin{tabular}{|c|c|}
\hline $\begin{array}{c}\text { Données expérimentales } \\
\text { et estimations }\end{array}$ & $\begin{array}{c}\text { Résultats } \\
\text { des calculs }\end{array}$ \\
\hline Antipov 2003: Interférométrie transitoire (pompe à & \\
\hline $808 \mathrm{~nm}$, sonde at $633 \mathrm{~nm}$ & \\
$\Delta \alpha_{4 \mathrm{~F}-41}(633 \mathrm{~nm}) \approx 4.0 \times 10^{-26} \mathrm{~cm}^{3}$ & $\Delta \alpha_{4 \mathrm{~F}-41}(633 \mathrm{~nm}) \approx 2.0 \times 10^{-26} \mathrm{~cm}^{3}$ \\
Estimation à $1064 \mathrm{~nm}:$ & \\
$\Delta \alpha_{4 \mathrm{~F}-41}(1064 \mathrm{~nm}) \approx 3.3 \times 10^{-26} \mathrm{~cm}^{3}$ & $\Delta \alpha_{4 \mathrm{~F}-41}(1064 \mathrm{~nm}) \approx 2.0 \times 10^{-26} \mathrm{~cm}^{3}$ \\
$\Delta \alpha_{2 \mathrm{~F}(2)-41}(633 \mathrm{~nm}) \approx 1.5 \times 10^{-26} \mathrm{~cm}^{3}$ & $\Delta \alpha_{4 \mathrm{~F}(2)-41(633 \mathrm{~nm}) \approx 2.0 \times 10^{-25} \mathrm{~cm}^{3}}$ \\
\hline (modifié en accord à l'aide de nos propres données) & \\
\hline$\frac{\text { Powell 1989: mélange 4-ondes à } 514 \mathrm{~nm}}{\Delta \alpha_{4 \mathrm{~F}-41}(514 \mathrm{~nm}) \approx 4.9 \times 10^{-26} \mathrm{~cm}^{3}}$ & $\Delta \alpha_{4 \mathrm{~F}-41}(514 \mathrm{~nm}) \approx 2.0 \times 10^{-26} \mathrm{~cm}^{3}$ \\
\hline
\end{tabular}

mentionnée plus haut, l'augmentation des variations d'indice observées en présence d'un pompage simultané à 808 et $266 \mathrm{~nm}$.

Ces résultats théoriques et expérimentaux différent néanmoins d'un facteur 1.5 à 2 . Bien que nous n'ayons pas encore de certitude, il semblerait que les valeurs expérimentales obtenues pourraient être supérieures aux valeurs théoriques en raison d'une contribution supplémentaire provenant de bandes de transfert de charge métal-ligand, donc, dans le cas du YAG :Nd, de transfert de charge $\mathrm{Nd}^{3+} \rightarrow \mathrm{O}^{2-}$. Ce type de contribution a déjà été invoqué dans le passé dans le cas des matériaux dopés par l'ion $\mathrm{Cr}^{3+}$, notamment dans le cas de $\mathrm{LiCaAlF}_{6}: \mathrm{Cr}$. Il est très difficile d'avancer dans cet hypothèse dans le cas de l'ion $\mathrm{Nd}^{3+}$, les bandes d'absorption entre configurations électroniques fondamentale et excitée $4 \mathrm{f}^{3}$ et $4 \mathrm{f}^{2} 5 \mathrm{~d}$ à l'origine des variations d'indice de réfraction invoquées précédemment se situant à plus basse énergie que les bandes de transfert de charge mentionnées plus haut.

Cette piste reste néanmoins très sérieuse car il semble bien par exemple que ce soit ce type de bande de transfert de charge qui soit à l'origine des fortes variations d'indice de réfraction observées récemment dans le cas du YAG : $\mathrm{Yb}$ [31], un autre matériau laser très important pour l'application laser de très forte puissance. Dans le cas du YAG :Yb, en effet, les bandes d'absorption entre configurations électroniques fondamentale et excitée $4 \mathrm{f}^{13}$ et $4 \mathrm{f}^{12} 5 \mathrm{~d}$ qui pourraient être à l'origine des variations d'indice de réfraction observées se situent à bien plus haute énergie que la bande de transfert de charge $\mathrm{Yb}^{3+} \rightarrow \mathrm{O}^{2}$ (voir Figure 4). En effet, tandis que les premières se situent au delà de $150 \mathrm{~nm}$ $\left(67000 \mathrm{~cm}^{-1}\right)$, il a été démontré expérimentalement [32] que la seconde commençait dès $230 \mathrm{~nm}$, avec un premier maximum vers $210 \mathrm{~nm}$. La position de cette bande de transfert de charge peut être d'ailleurs déterminée approximativement en utilisant la relation bien connue de K. Jorgensen [21], $E_{g s a}^{L M C T} \approx$ 30000. $\left[\chi\left(\mathrm{O}^{2-}\right)-\chi\left(\mathrm{Yb}^{3+}\right)\right]$, où les $\chi$ représentent l'électronégativité des ions considérés, donc $\mathrm{Yb}^{3} \mathrm{et}$ $\mathrm{O}^{2}$, ce qui conduit, avec $\chi\left(Y b^{3+}\right)=1.68$ et $\chi\left(O^{2-}\right)=3.2$, à $E_{\mathrm{gsa}}^{\mathrm{LMCT}} \approx 45600 \mathrm{~cm}^{-1}$ soit $220 \mathrm{~nm}$, donc très proche de la valeur expérimentale.

Partant de cette valeur, cela signifie que l'on doit s'attendre à l'existence d'une bande d'absorption entre état excité émetteur ${ }^{2} \mathrm{~F}_{5 / 2}$ de l'ion $\mathrm{Yb}^{3+}$ (situé vers $10300 \mathrm{~cm}^{-1}$ ) et ion $\mathrm{O}^{2-}$ aux alentours de $47600-10300=35300 \mathrm{~cm}^{-1}$, donc vers $280 \mathrm{~nm}$. Or c'est exactement ce que nous avons observé (voir Figure 5) en pompant dans le niveau ${ }^{2} \mathrm{~F}_{5 / 2}$ du $\mathrm{YAG}: \mathrm{Yb}$ avec un laser à impulsion à $980 \mathrm{~nm}$ et en sondant la transmission du matériau dans le proche UV entre 200 et $400 \mathrm{~nm}$.

La contribution des bandes de transfert de charge dans les variations d'indice de réfraction observées dans les matériaux laser du type YAG :Yb et YAG :Nd ne fait donc aucun doute. Reste à savoir maintenant l'importance de cette nouvelle contribution. Des expériences complémentaires 


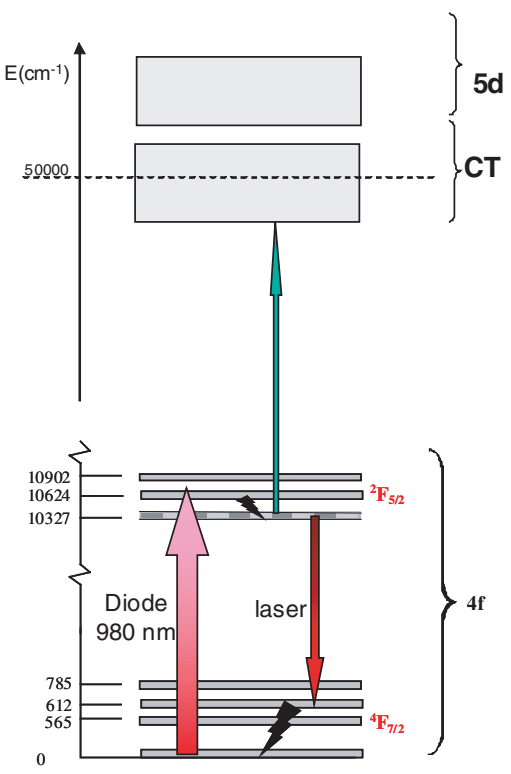

Figure 5. Niveaux d'énergie des l'ion $\mathrm{Yb}^{3+}$ dans YAG.

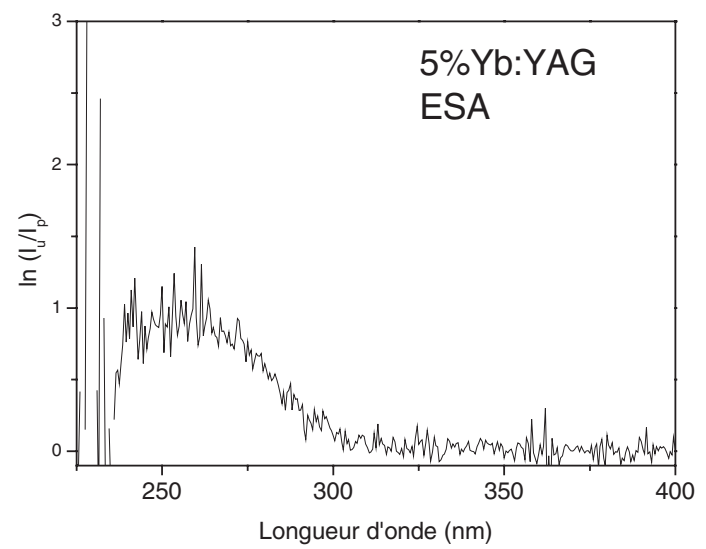

Figure 6. Spectre d'absorption dans l'état excité (ESA) de l'ion $\mathrm{Yb}^{3+}$ dans YAG enregistré dans le proche UV en pompant le matériau á $980 \mathrm{~nm}$.

d'interférométrie transitoire sont d'ores et déjà mises en œuvre pour approfondir cette question dans le cas du YAG :Yb et du YAG :Nd mais aussi pour en explorer les effets dans d'autres types de matrices laser importantes tels que $\mathrm{YVO}_{4}$ et $\mathrm{KYW}$.

\section{CONCLUSION}

Dans cette présentation, nous avons montré qu'il est maintenant possible de comprendre, connaissant la position des niveaux d'énergie des ions de terre rare par rapport aux bandes de conduction et de valence des réseaux hôtes, dans quelles circonstances un système donné peut conduire ou non à un effet laser dans le proche UV. Il a été montré ensuite, dans le cas des matériaux dopés terres rares pour lasers UV, que la connaissance expérimentale et théorique des spectres d'absorption à partir des états fondamental et excités de ces ions dans leur configuration électronique fondamentale $4 \mathrm{f}^{\mathrm{n}}$ vers des niveaux d'énergie supérieurs appartenant à leur première configuration électronique excitée $4 \mathrm{f}^{\mathrm{n}-1} 5 \mathrm{~d}$ permettait d'envisager des schémas de pompage optique par étapes utilisant des photons moins énergétiques, donc moins agressifs en terme de solarisation, que dans le cas d'un pompage direct. Nous avons montré enfin que la connaissance de ces spectres permettait également d'expliquer les variations d'indice de réfraction athermiques observées dans certains matériaux lorsqu'ils sont soumis à de fortes densités d'excitation et qu'une partie de ces variations d'indice pourrait être associée à l'existence et la proximité de bandes de transfert de charge « ligand-métal » très intenses. Des travaux sont en cours pour examiner cette hypothèse dans le cas de l'ion $\mathrm{Yb}^{3+}$ dans différentes matrices cristallines.

\section{Remerciements}

La majeure partie de ce travail a été effectuée au sein de l'équipe "Matériaux et Instrumentation Laser" du laboratoire CIRIL, d'abord dans le cadre d'un contrat avec l' «European Research Office of the US Army » à 
Londres, puis d'une «Action Coordonnée Optique » et d'un poste de chercheur invité du CNRS et enfin dans le cadre d'un contrat de collaboration INTAS, en particulier avec le Dr. O. Antipov de l'Institute of Applied Physics de Nizhny Novgorod (Russie). Une partie importante a été réalisée également en étroite collaboration avec le LPCML à Lyon. J'adresse donc tous mes remerciements aux thèsards (M. Laroche, S. Nicolas, A. Collombet, P. Cordelier), post-doctorant (P. Nagtegaele) et chercheurs (J. Margerie, Y. Guyot, M.F. Joubert, S. Girard, J.L. Doualan) et organismes (EOARD, CNRS, INTAS) concernés.

\section{Références}

[1] M. Laroche, J.L. Doualan, S. Girard, J. Margerie, R. Moncorgé, J. Opt. Soc. Am. B 17 (2000) 1291-1303

[2] M.F. Reid, L. van Pieterson, R.T. Wegh, A. Meijerink, Phys. Rev. B 62 (2000) 14744-14749

[3] A. Collombet, Y. Guyot, M.F. Joubert, M. Laroche, J. Margerie, R. Moncorgé, Phys. Rev. B 68 (2003) 035115(1-11)

[4] A.J.S. McGonigle, D.W. Coutts, S. Girard, R. Moncorgé, Opt. Comm. 193 (2001) 232

[5] A.J.S. McGonigle, R. Moncorgé, D.W. Coutts, Appl. Opt. 40 (2001) 4326-4333

[6] Z. Liu, T. Koseki, Y. Suzuki, N. Sarukura, K. Shimamura, T. Fukuda, M. Hirano, H. Hosono, Opt. Lett. 26 (5) (2001) 301-303

[7] B.C. Weber, A. Hirth, Opt. Comm. 128 (1996) 158-165

[8] 5. N. Passilly, M . Fromager, K. Ait-Ameur, R. Moncorgé, J. L. Doualan, A. Hirth, G. Quarles, J. Opt. Soc. Am. B 21 (2004) 531-538

[9] N. Passilly, E. Haouas, V. Ménard, R. Moncorgé, K. Aitameur, Opt. Comm. 260 (2006) 703-707

[10] O.L Antipov, A.S. Kuzhelev, D.V. Chausov, A.P. Zinov'ev, J. Opt. Soc. Am. B16 (1999) 1072-1079

[11] O.L Antipov, O.N. Eremeykin, A.P. Savikin, V.A. Vorob’ev, D.V. Bredikhin, M.S. Kuznetsov, IEEE J. Quant. Electr. 39 (2003) 910

[12] V.V. Semashko, M.A. Dubinskii, R. Yu. Abdulsabirov, S.L. Korableva, A.K. Naumov, A.S. Nizamutdinov, M.S. Zhuchkov, SPIE Proceed. XI Int. Feofilov Symp. (Kazan, Russia, sept. 24-28, 2001) vol. 4766 (2002)

[13] Z. Liu, K. Shimamura, K. Nakano, N. Mujilatu, T. Fukuda, T. Koseki, H. Ohtake, N. Sarukura, J. Appl. Phys. 39 (2000) L466

[14] N. Sarukura, Z. Liu, H. Ohtake, Y. Segawa, M.A. Dubinskii, R.Y. Abdulsabirov, S.L. Korableva, A.K. Naumov, V.V. Semashko, Opt. Lett. 22 (1997) 994

[15] M.A. Dubinskii, A.C. Cefalas, J. Opt. Soc. Am. B9 (1992) 1148

[16] R.W. Waynant, Ph. H. Klein, Appl. Phys. Lett. 46 (1985) 14

[17] C.W. Thiel, H. Crugel, H. Wu, Y. Sun, G.J. Lapeyre, R.L. Cone, R.W. Equall, R.M. MacFarlane, Phys. Rev. B 64 (2001) 085107-1-13

[18] J. Sugar, J. Reader, J. Chem. Phys. 59 (1973) 2093-2089

[19] R.D. Shannon, Acta Cryst. A 32 (1976) 751

[20] O. Guillot-Noel, B. Bellamy, V. Viana, D. Gourier, Phys. Rev. B60 (1999) 1668-1677

[21] R. Moncorgé in «Ultraviolet Spectroscopy and UV lasers » Chap. 9, P. Misra, M.A. Dubinskii (eds) Marcel Dekker, New York, 2002

[22] C.W. Thiel, H. Crugel, Y. Sun, G.J. Lapeyre, R.M. MacFarlane, R.W. Equall, R.L. Cone, J. Lumin. 94-95 (2001) 1-6

[23] L. Pieterson, M.F. Reid, G.W. Burdick, A. Meijerink, Phys. Rev. B 65 (2002) 045114-1-13

[24] P. Dorenbos, Phys. Rev. B 62 (2000) 15640, J. Lumin. 111 (2005) 89-104

[25] S. Jobic (IMN Nantes) communication privée

[26] M. Laroche, A. Braud, S. Girard, J.L. Doualan, R. Moncorgé, M. Thuau, L.D. Merkle ; J. Opt. Soc. Am.B 16 (1999) pp 2269-2277 
[27] P. Segonds, B. Boulanger, B. Ménaert, J. Zaccaro, J.P. Salvestrini, M.D. Fontana, R. Moncorgé, F. Porée, G. Gadret, J. Mangin, A. Brenier, G. Boulon, G. Aka, D. Pelenc, Opt. Mat. (2006) sous presse

[28] P. Sillard, A. Brignon, J.P. Huignard, IEEE J. Quant. Electron. QE 34 (1998) 465

[29] O. N. Eremeykin, O. L. Antipov, A. Minassian, M. J. Damzen, Opt. Lett. 29 (20) (2004) 23902392

[30] R.C. Powell, S. A. Payne, L. L. Chase, G. D. Wilke, Opt. Lett. 14 (21) (1989) 1204-1206

[31] O.L. Antipov, D.V. Bredikhin, O.N. Eremeykin, A.P. Savikin, E.V Ivakin, A.V. Sukhadolau, Opt. Lett. 31 (2006) 763-765

[32] L. van Pieterson, M. Heeroma, E. de Heer and A. Meijerink, J. Lumin. 91 (2000) 177-193 